\title{
Visite dans les rouages du climat
}

Jean Poitou (jean.poitou@wanadoo.fr)

Ancien adjoint au directeur du Laboratoire des Sciences du Climat et de l'Environnement (CEA/CNRS)

\section{La machine climatique}

est une machine thermique

qui reçoit sa chaleur

du Soleil, et qui la redistribue

à la surface du globe terrestre

par la circulation couplée

des deux fluides, atmosphère

et océan, en interaction avec

I'environnement. Le cycle de

l'eau, présente sur Terre sous

ses trois formes physiques :

solide, liquide et gazeuse,

y joue un rôle capital.

Les forçages externes

induisent des modifications

du climat qui sont fortement

modulées par des rétroactions

de l'environnement.

Pour élucider le fonctionnement

d'un tel système et étudier

ses devenirs possibles, toute

une panoplie de modèles

a dû être mise en œuvre.
Le mot « climat » évoque généralement les conditions météorologiques : températures, pluviosité, systèmes de vents... et c'est ce qu'historiquement la climatologie s'est d'abord attachée à décrire. Une autre façon de définir un climat est de se référer à la biosphère qui s'y développe spontanément : la végétation, qui a longtemps servi à définir les climats, mais aussi toute la faune qui y prospère. En parallèle, les sciences physiques ont permis de décrypter le fonctionnement de la machine climatique, puis de la modéliser [1]. Cette machine fait interagir de nombreux processus au sein des enveloppes extérieures de la Terre.

Écrit à l'occasion de la conférence sur les changements climatiques (COP21) qui s'est tenue à Paris en décembre 2015, cet article présente les principaux acteurs du climat et, sur des exemples représentatifs, fait prendre conscience au lecteur des multiples interactions à l'œuvre dans les processus climatiques. Pour une description complète de la machine climatique, le lecteur est renvoyé aux ouvrages cités en références [2-5].

La machine climatique est une machine thermique, qui reçoit son énergie du Soleil (le flux géothermique, 0,03\% du flux solaire absorbé par la Terre, est négligeable). Son fonctionnement fait interagir à des degrés divers toutes les composantes de l'environnement superficiel de notre planète.

La Terre (atmosphère et surface) renvoie vers l'espace $30 \%$ de l'énergie reçue du Soleil. Le reste, $70 \%$, est absorbé et transformé en chaleur. Cette chaleur en excès est évacuée vers l'espace sous forme de rayonnement électromagnétique, seule forme d'énergie susceptible de partir dans le vide interplanétaire. Du fait de la sphéricité de la Terre, la quantité d'énergie reçue du Soleil est distribuée inégalement à la surface du globe, maximale près de l'équateur. En un point donné, la température serait stable en moyenne si la quantité d'énergie qui
Bilan radiatif au sommet de l'atmosphère

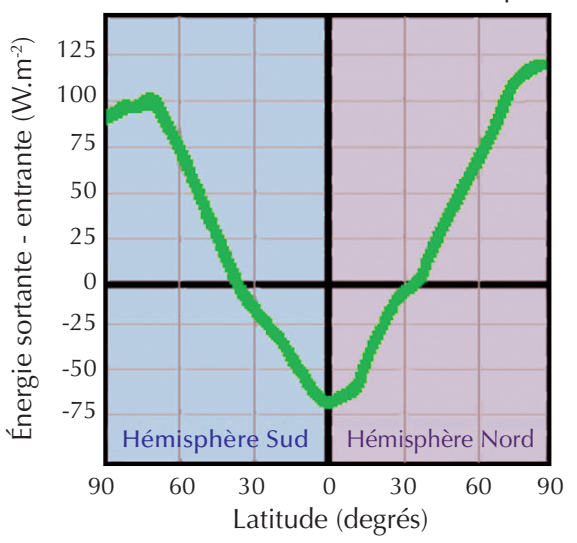

1. Écart en fonction de la latitude, entre l'énergie qui quitte la Terre et l'énergie solaire incidente (mesures du satellite ERBE, effectuées entre 1984 et 2003). Sauf aux latitudes voisines de $30^{\circ}$ nord ou sud, le bilan est déséquilibré. l'équateur ne renvoie vers l'espace qu'une fraction de ce qu'il en reçoit, et inversement pour les hautes latitudes.

part vers l'espace était égale à la quantité d'énergie solaire qui arrive. Les observations par satellites montrent (fig. 1) que ce bilan radiatif est excédentaire dans les régions tropicales, et est déficitaire dans les régions polaires ; il y a donc un transport d'énergie des basses latitudes vers les hautes latitudes. Les deux fluides de l'enveloppe terrestre, l'atmosphère et l'océan, y contribuent chacun environ pour moitié en moyenne globale.

\section{Le transport de chaleur}

Le rayonnement solaire est absorbé par le sol sur les continents, dans les premiers mètres sous la surface dans l'océan. La chaleur est transmise à la troposphère par le bas, alors que l'océan est chauffé par le haut, donnant à ces deux fluides une répartition verticale de température très différente (fig. 2). Les transports verticaux 


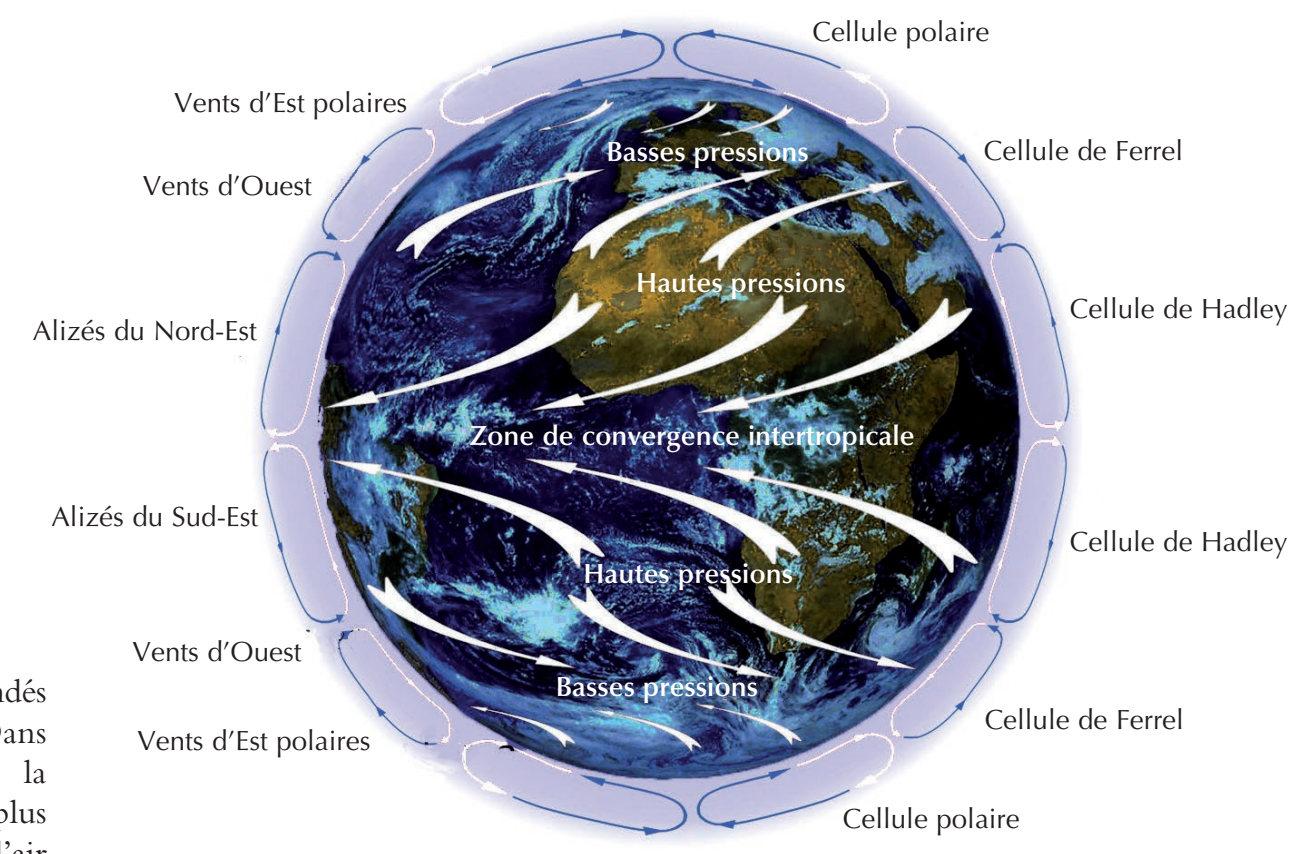

de matière sont essentiellement commandés par la densité locale du fluide. Dans l'atmosphère chauffée par le bas, la convection assure l'ascension d'air plus chaud (et donc moins dense) que l'air environnant, et la descente de l'air froid. Dans l'océan, les gradients de densité dépendent de la température et de la salinité : l'eau est généralement plus chaude à la surface qu'en profondeur; sous la glace de mer, le rejet de saumure par la glace (dans laquelle le sel est pratiquement insoluble) augmente la densité de l'eau et provoque sa plongée.

Le transport vertical au sein de l'atmosphère génère des variations de pression qui provoquent les déplacements horizontaux d'air, les vents. Le mouvement se ferait simplement des hautes pressions vers les basses pressions si la Terre n'était pas en rotation sur elle-même. La force de

4. Les grandes tendances du système global des vents (flèches blanches). Le déséquilibre thermique a pour conséquence la formation de cellules de convection, telles que les cellules de Hadley près de l'équateur.

Coriolis dévie les mouvements et donc les vents vers la droite dans l'hémisphère nord, vers la gauche dans l'hémisphère sud, les faisant tourner autour des dépressions, ce qu'on appelle la circulation géostrophique (fig. 3) [6]. Le système global des vents est schématisé sur la figure 4.

Les vents sont affectés par le relief et la rugosité des régions qu'ils traversent. À l'interface air-océan, un échange de

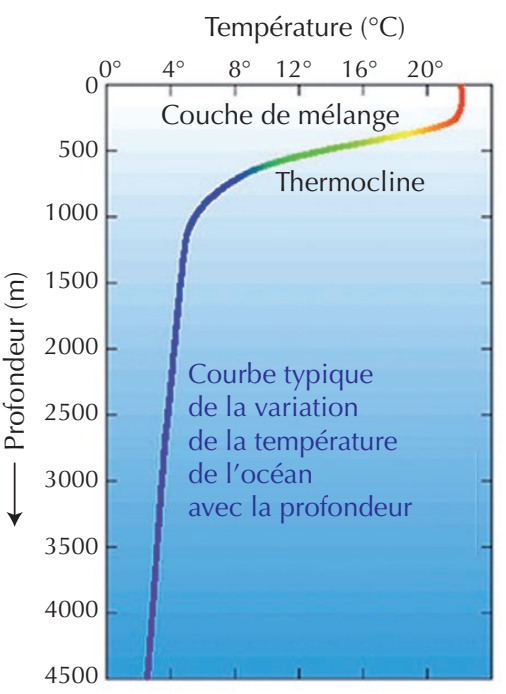

La mer est chauffée par le haut

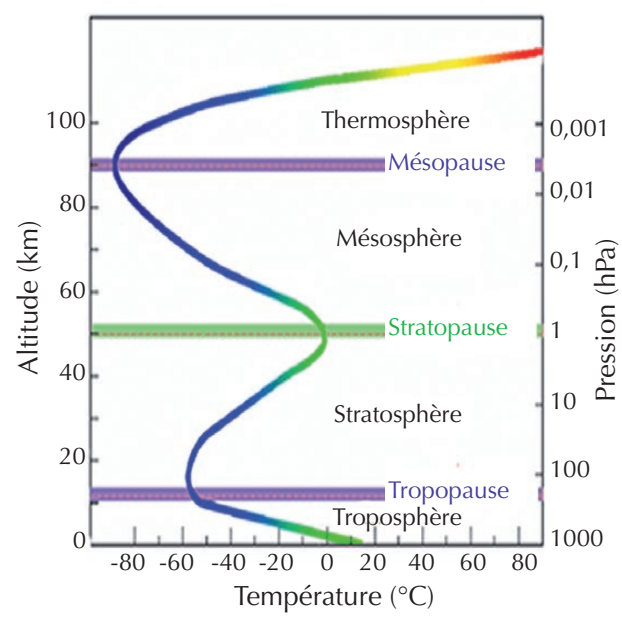

La troposphère est chauffée par le bas

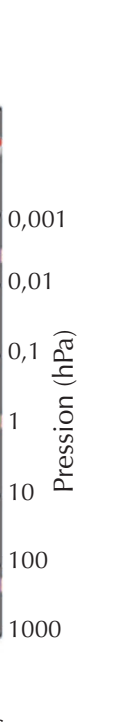

2. Profil vertical de température typique pour l'atmosphère (à gauche) et pour l'océan (à droite). La troposphère, qui contient l'essentiel de la masse de l'atmosphère dans les dix premiers kilomètres, est chauffée par le bas, tandis que l'océan est chauffé par le haut. Dans l'océan, la thermocline est d'amplitude très variable selon la région concernée.

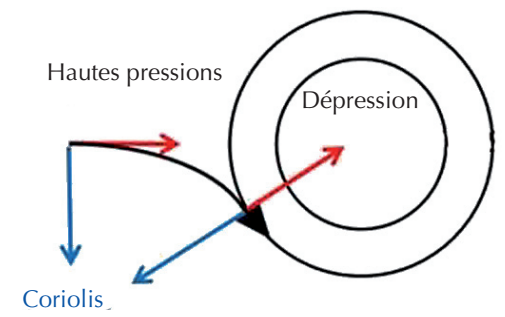

3. Circulation géostrophique des vents dans l'hémisphère nord. La force due au gradient de pression (en rouge) est équilibrée par la force de Coriolis (en bleu). Le vent (flèche noire) suit les isobares (cercles) autour de la dépression.

quantité de mouvement entre les deux fluides donne naissance aux courants marins de surface (jusqu'à environ 1000 mètres de profondeur). Soumis aux forces de Coriolis, ces courants décrivent de vastes boucles au sein des grands bassins, les "gyres "; le Gulf Stream en est un exemple.

L'océan mondial connait aussi une circulation profonde, la circulation thermohaline, dont le moteur, la densité, dépend de la température et de la salinité de l'eau. L'eau qui arrive aux hautes latitudes des régions polaires, a vu sa salinité croître par évaporation et a cédé de sa chaleur à l'atmosphère par rayonnement infrarouge, chaleur sensible (échangée sans transition de phase) et évaporation. L'eau salée qui gèle est très froide $\left(-1,8^{\circ} \mathrm{C}\right.$ pour une salinité de $35 \mathrm{~g} /$ litre). À l'interface glaceeau, le sel tend à migrer de la phase solide vers la phase liquide, rejetant des saumures qui renforcent la salinité de l'eau. L'eau 


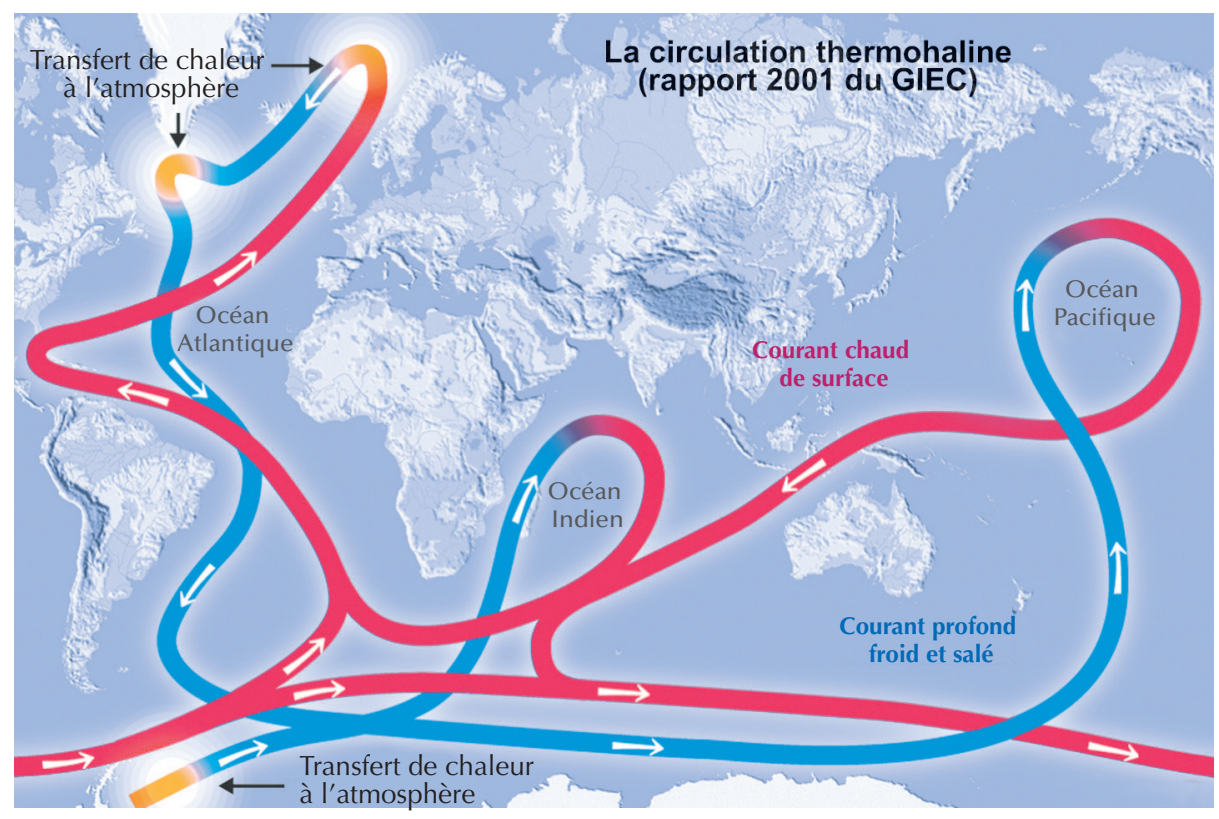

5. Schéma du « tapis roulant » de la circulation thermohaline. En bleu, la circulation profonde d'eau froide : en rouge, la circulation de surface d'eau chaude. Dans les zones de plongée, l'océan apporte de la chaleur à l'atmosphère.

\section{El Niño - La Niña}

encadré

Dans le Pacifique équatorial, les hautes pressions se situent normalement à l'est, sur les côtes du Pérou, les basses pressions à l'ouest, sur les Philippines. Les alizés (fig. 4) provoquent une forte évaporation, à l'origine d'une activité convective et de précipitations soutenues sur l'ouest du bassin, où s'accumulent les eaux chaudes de surface.

Tous les 3 à 7 ans, les alizés faiblissent, l'eau chaude reflue vers l'est, la zone de convection active migre vers l'est, pouvant provoquer des précipitations intenses sur le Pérou et une grande sécheresse aux Philippines. C'est un événement El Niño, ainsi nommé parce qu'il atteint son paroxysme vers Noël. Les effets d'un fort El Niño débordent largement du Pacifique intertropical, affectant l'océan Indien à l'ouest et jusqu'au bord ouest de l'Atlantique à l'est. Un événement El Niño est souvent suivi du phénomène inverse, la Niña, qui se manifeste par des alizés plus forts qu'à l'ordinaire et un amenuisement de la zone chaude des eaux de surface du Pacifique équatorial.

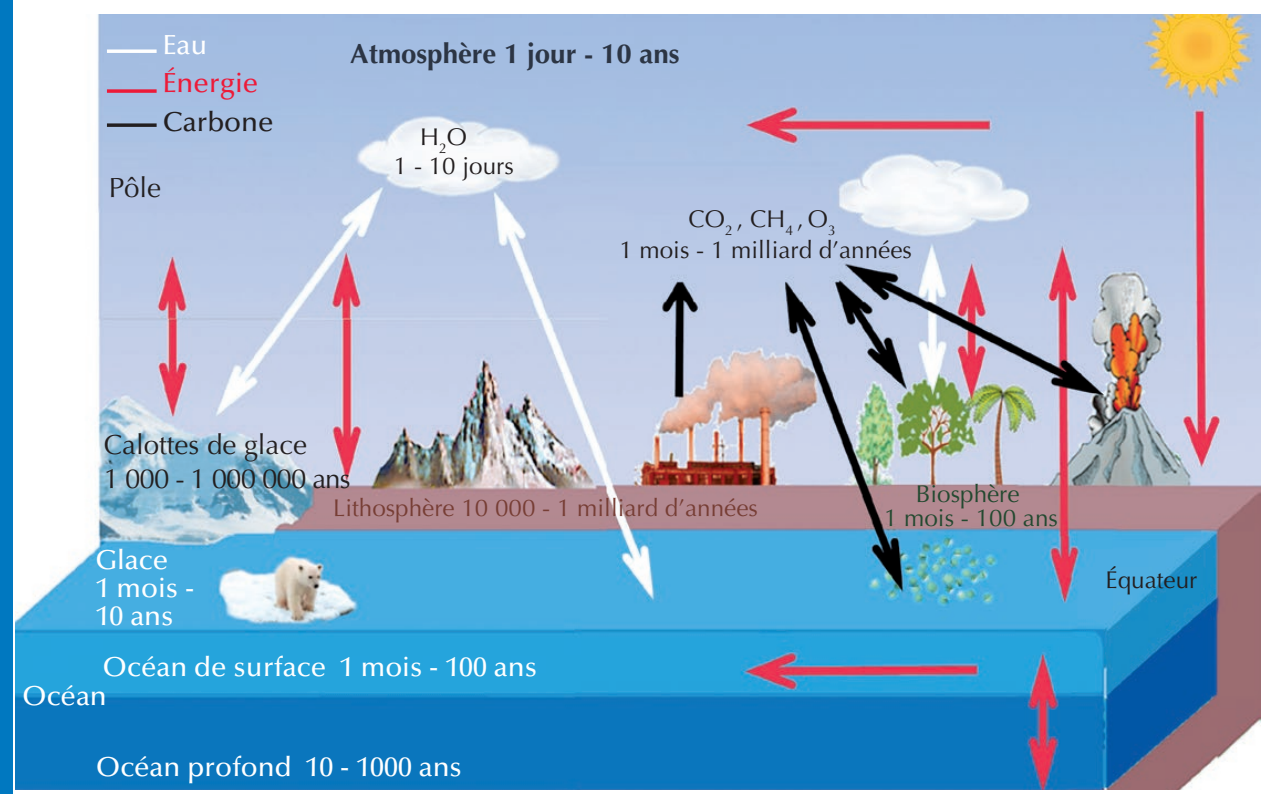

6. Les échanges d'énergie, de vapeur d'eau, de gaz à effet de serre ont des temps caractéristiques très divers, allant de la journée au milliard d'années, selon les milieux et processus en jeu.
\〉

très dense plonge jusqu'au fond de l'océan où elle entreprend un long périple qui lui fera parcourir l'ensemble des océans du monde avant de remonter lentement. Alors, reprise par les courants de surface, elle est finalement ramenée là où elle a plongé, après un voyage qui aura pu durer jusqu'à un millénaire. C'est ce qu'on a nommé le «tapis roulant » de la circulation océanique (fig. 5). Ainsi, la plongée des eaux dans le grand nord est le moteur de la remontée des eaux chaudes du Gulf Stream vers le nord de l'Atlantique, via la "dérive nord-atlantique".

Les vents jouent aussi un rôle moteur dans la remontée des eaux froides vers la surface ("upwelling") sur les franges continentales ou dans la zone de convergence intertropicale.

Le fonctionnement du climat fait donc intervenir de façon étroitement couplée les deux fluides qui enveloppent la Terre. À leur interface, ils échangent de la chaleur (sensible et surtout latente), de la quantité de mouvement à l'origine des courants marins de surface, et de l'eau sous forme gazeuse ou liquide. Les océans fournissent aussi à l'atmosphère des particules de sels minéraux dans les embruns, et des composés soufrés (émis par le plancton), dont l'oxydation en sulfates va produire des noyaux de condensation indispensables à la formation des nuages. Les temps de réponse caractéristiques de l'atmosphère et de l'océan sont très divers (fig. 6), ce qui en fait un système couplé complexe, sujet à des oscillations propres, dont la plus connue est l'oscillation australe El NiñoLa Niña (voir encadré 1).

\section{L'eau sous toutes ses formes}

L'eau dans ses trois phases joue un rôle essentiel dans les bilans d'énergie et le fonctionnement du climat (voir l'encadré 2).

- L'évaporation, qui se produit essentiellement sur les océans, en refroidit la surface, la vapeur d'eau apportant à l'atmosphère sa chaleur latente de condensation. Transportée par les masses d'air humide, cette chaleur est restituée à l'atmosphère là où la vapeur se condense après un délai moyen d'une quinzaine de jours. La chaleur ainsi libérée entretient des convections importantes, qui alimentent les dépressions. Elle intervient dans la formation des grands cumulonimbus d'orage. Elle fournit leur énergie aux 

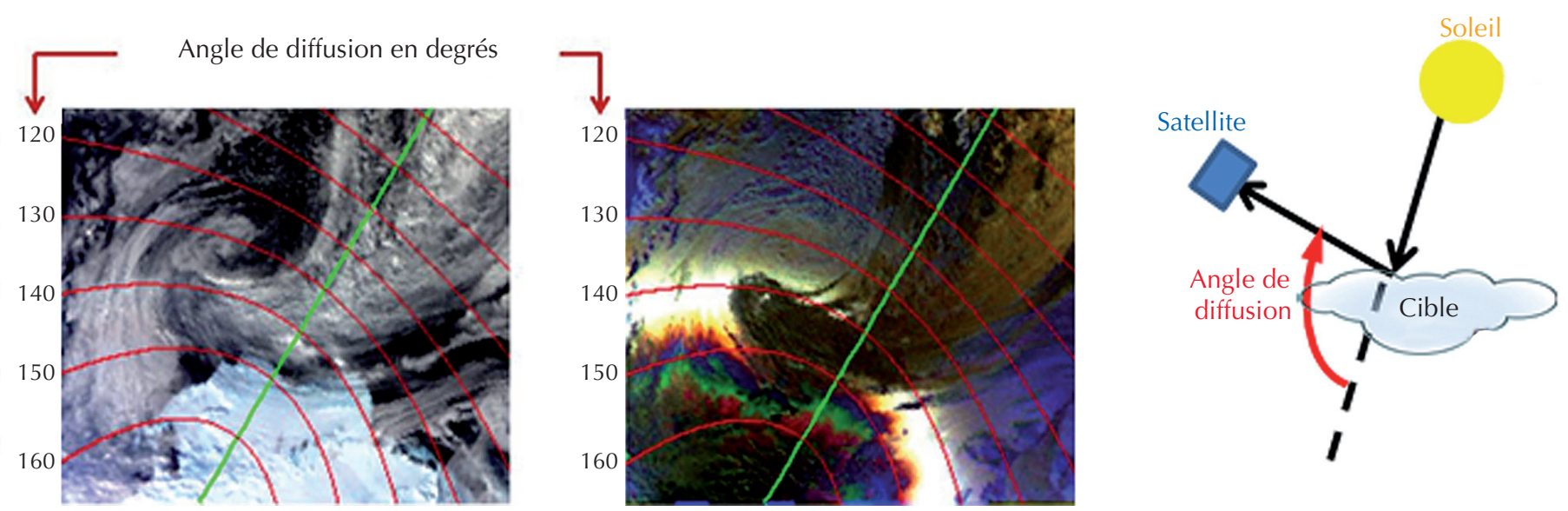

7. Observation de la lumière réfléchie par les nuages. La même scène au-dessus de l’Antarctique en lumière naturelle (à gauche) et en lumière polarisée (au centre), mesurée par le radiomètre spatial POLDER du microsatellite français PARASOL (2004-2013). Le principe de la mesure est indiqué sur le schéma de droite. Parmi les nuages visibles sur l'image de gauche, certains, les nuages d'eau ont, en lumière polarisée, un effet d'arc-en-ciel. Ils sont partiellement masqués par la présence à plus haute altitude de nuages de glace, qui ne polarisent pas la lumière.

tempêtes tropicales (ouragans, typhons, cyclones). C'est aussi la convection, liée à la condensation avec précipitations intenses dans la zone de convergence intertropicale, qui provoque la forte ascendance de l'atmosphère à l'origine des cellules de Hadley (fig. 4).

- La vapeur d'eau, présente surtout dans la basse troposphère, est responsable de 50\% de l'effet de serre naturel. La quantité de vapeur d'eau que peut contenir l'atmosphère augmente avec la température (en vertu de la relation de ClausiusClapeyron), à raison $\mathrm{de} \approx 7 \% \mathrm{par}{ }^{\circ} \mathrm{C}$ à nos températures.

- Les nuages bas sont formés d'eau liquide (fig. 7). Ils font écran au rayonnement solaire incident (effet parasol). Les nuages élevés et fins, les cirrus, sont constitués de glace. Absorbant les infrarouges, ils contribuent au confinement de la chaleur dans la troposphère.

- Sous forme solide, l'eau se trouve dans les nuages élevés, dans les précipitations sous forme de neige ou de grêle, et dans la cryosphère : glace de mer, glaciers continentaux et alpins, pergélisol, neige sur le sol. Du fait de leur pouvoir réfléchissant (l'albédo) très élevé (jusqu'à 90\%), les surfaces enneigées ou englacées limitent fortement l'absorption locale du rayonnement solaire. Le seuil liquideglace $\left(0^{\circ} \mathrm{C}\right.$ pour l'eau douce) est souvent franchi par l'eau à la surface du globe.

\section{Le cycle de l'eau}

encadré 2

La Terre contient 1400 millions de $\mathrm{km}^{3}$ d'eau : environ 2,2\% sous forme de glace, essentiellement sur l'Antarctique, 95\% dans les océans, de $1 \%$ à $2 \%$ dans les eaux souterraines, le reste dans les lacs, rivières, mers intérieures, cellules des organismes vivants. L'atmosphère n'en a que $0,001 \%$.

L'océan évapore $410000 \mathrm{~km}^{3}$ d'eau par an. La vapeur d'eau parcourt environ $1000 \mathrm{~km}$ dans l'atmosphère en 15 jours, avant de se condenser pour donner des nuages. Les gouttes d'eau ou les cristaux y croissent par coalescence jusqu'à ce que, leur poids dépassant la résistance de l'air, ils tombent en précipitations. $110000 \mathrm{~km}^{3}$ d'eau tombent sur les continents d'où s'en évaporent (ou se subliment) $70000 \mathrm{~km}^{3}$ soit directement, soit par la transpiration de la végétation. Le reste, $40000 \mathrm{~km}^{3}$, ruisselle ou s'infiltre dans le sol avant de rejoindre les océans par les fleuves ou par les écoulements souterrains ou, pour environ $1200 \mathrm{~km}^{3}$, par la fonte des glaciers et des icebergs. Au total, $370000 \mathrm{~km}^{3}$ retombent dans l'océan sous forme de précipitations.

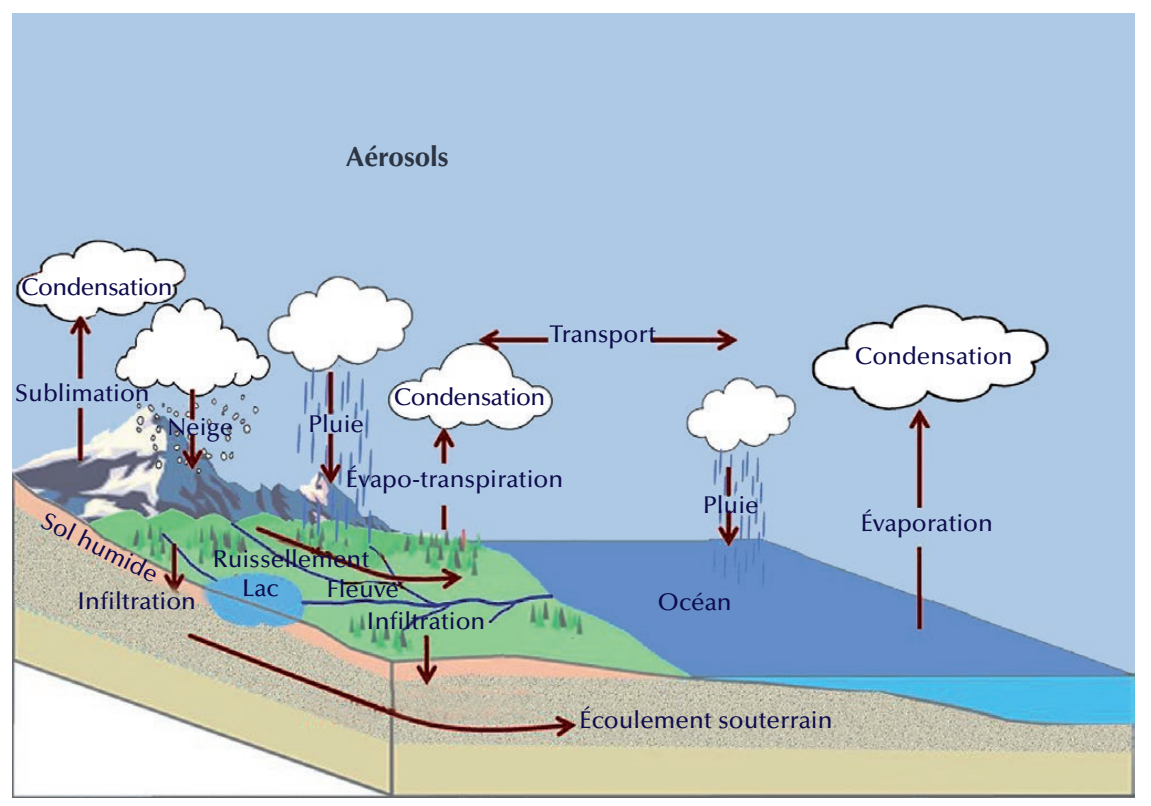

E1. Représentation schématique du cycle de l'eau sur la Terre. 
\〉

Lors du franchissement de ce seuil, la variation considérable de l'albédo produit un comportement non linéaire du climat, les effets étant bien plus importants que la petite variation initiale de température.

\section{Interactions climat-environnement}

Le climat de la Terre dépend de son bilan radiatif : tout ce qui est susceptible de modifier le flux absorbé ou le flux sortant va perturber le climat terrestre.

- Le flux d'énergie arrivant à la surface dépend en premier lieu du flux solaire atteignant la Terre. Ce flux peut changer par suite des variations de l'activité du Soleil. Il oscille aussi à l'échelle des dizaines de millénaires, avec l'éloignement de la Terre par rapport au Soleil, du fait des fluctuations quasi cycliques de ses paramètres astronomiques : obliquité, précession, excentricité de l'orbite.

- Dans le domaine visible et le proche infrarouge, l'atmosphère est essentiellement transparente pour le rayonnement solaire. La majeure part de l'absorption est le fait d'aérosols, tels ceux de noir de carbone. Mais les rayons solaires sont aussi diffusés par les molécules de l'atmosphère et les particules des aérosols, et réfléchis par les nuages dont l'importance, la longévité et les propriétés optiques sont elles-mêmes dépendantes des aérosols.

- Le pouvoir de réflexion du rayonnement incident par la surface (albédo) dépend de la nature de cette dernière. Une surface de neige ou de glace a un albédo élevé, 60 à $90 \%$. La présence de végétation réduit l'albédo, qui peut devenir très faible $(10 \%)$ dans le cas de la forêt équatoriale sombre. L'albédo des océans est aussi très faible. L'albédo planétaire, $30 \%$, est plus élevé que l'albédo moyen de la surface du fait de la présence des nuages.

- Dans le domaine infrarouge thermique, celui du flux sortant vers l'espace, l'atmosphère agit fortement sur le rayonnement. Ici interviennent de nouveau les nuages et les aérosols dans les processus de réflexion, de diffusion et d'absorption. Mais le processus majeur est l'absorption par les gaz dits "à effet de serre " (voir l'encadré 3), sans lesquels la vie sur Terre n'existerait pas.

Les continents sont des obstacles infranchissables pour la circulation océanique. Ils modulent la circulation atmosphérique

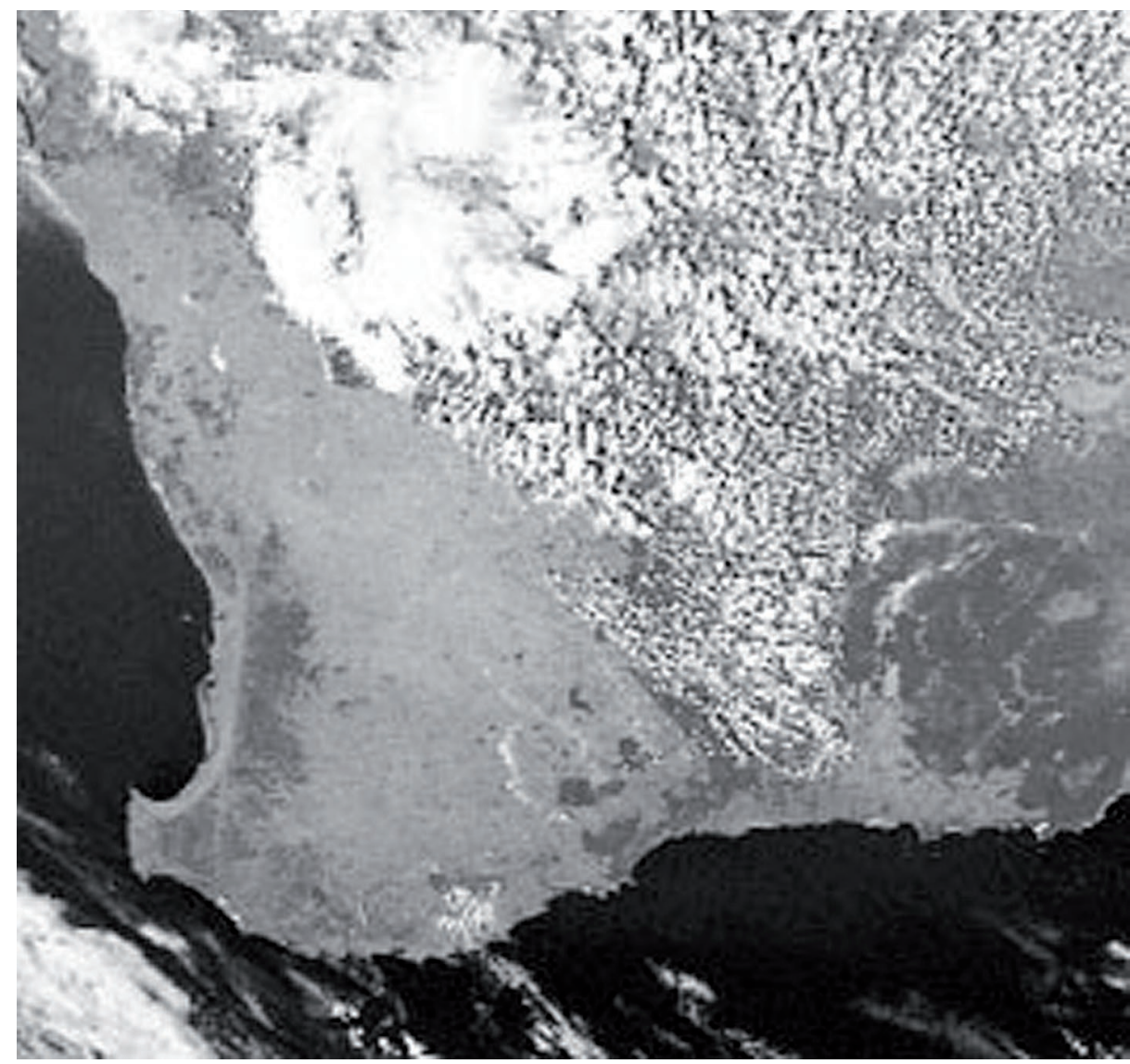

8. Vue par satellite en lumière visible du sud-ouest de l’Australie, le 3 janvier 1999. La limite entre la zone nuageuse et le ciel clair est aussi la limite entre la forêt naturelle et la zone des terres cultivées.

(Source : UCAR/NCAL/EOL ; www.nsst.uah.edu/ nair/BUFEX05/study_area.html).

tant horizontale que verticale : la présence de montagnes force les masses d'air à s'élever et donc à se refroidir, amenant éventuellement à la condensation de vapeur d'eau et à des précipitations, un effet particulièrement marqué en Asie, où l'Himalaya contribue fortement à l'ampleur des moussons humides.

\section{Rétroactions}

Le climat façonne les différentes facettes de l'environnement terrestre : cryosphère, types de végétation et de faune, érosion... Il détermine l'évaporation des eaux, la nébulosité, les précipitations, les soulèvements d'aérosols naturels (feux de forêt ou de savane, poussière des sols nus). Mais les modifications que ses évolutions infligent à l'environnement réagissent à leur tour sur le climat pour, selon les cas, les accentuer ou les inhiber, comme vont le montrer quelques exemples.

- L'élévation de la température au sol entraîne un accroissement de l'énergie rayonnée (loi de Stefan-Boltzmann), qui tempère ce réchauffement. De même, du fait du gradient de température atmosphérique, le réchauffement local de l'air va entraîner un mouvement de convection par lequel l'air chaud ascendant va être remplacé par de l'air descendant plus froid. Ce sont des rétroactions négatives.

- Les entrées en glaciation (encadré 4) mettent en jeu plusieurs rétroactions positives.

- Certains cas sont plus complexes : le réchauffement du climat accroît l'évaporation d'eau ; conformément à la loi de Clausius-Clapeyron, l'atmosphère pourra contenir une plus grande quantité de vapeur d'eau, ce qui accentuera l'effet de serre et renforcera donc le réchauffement. Mais on aura aussi une plus forte nébulosité. Si ce sont des nuages bas, leur effet sera de tempérer le réchauffement (effet parasol) ; par contre, si ce sont des nuages hauts, des cirrus, le réchauffement en sera renforcé.

- Un cas intéressant est celui de la végétation. Sa présence diminue l'albédo du sol, renforçant l'absorption des rayons 
Qu'appelle-t-on effet de serre?

En l'absence d'atmosphère, une Terre ayant un albédo planétaire de 30\% (celui de notre Terre actuelle) et recevant le même flux solaire incident de $1360 \mathrm{~W} \cdot \mathrm{m}^{-2}$ aurait, selon la loi de Stefan-Boltzmann, une température moyenne de $255 \mathrm{~K}$, soit $-18^{\circ} \mathrm{C}$. Or, la température moyenne au sol de notre planète est de $+15^{\circ} \mathrm{C}$.

Comme le montrent les calculs de transfert d'énergie dans l'atmosphère, cette différence entre la température radiative planétaire et la température au sol, attribuée à l' " effet de serre ", est due à l'action conjuguée de plusieurs processus qui incluent d'une part le transport vertical de chaleur à travers l'atmosphère par conduction, convection et rayonnement, et d'autre part la diffusion, l'absorption et l'émission du rayonnement infrarouge par les molécules de gaz de l'atmosphère. Ce phénomène d'effet de serre n'est possible que dans une atmosphère dont la température décroît avec l'altitude ${ }^{(*)}$. II fait intervenir la structure verticale de l'atmosphère et le fait que les photons concernés sont émis au sein de l'atmosphère, qui ne peut donc pas être traitée comme un absorbant mince. Le terme " effet de serre " se réfère au fait que l'atmosphère confine la chaleur et donc accroît la température à basse altitude, de même que la vitre d'une serre horticole confine, par un processus différent, la chaleur à l'intérieur de la serre.

L'effet de serre est un phénomène incontournable dans la compréhension du climat de la Terre mais aussi des autres planètes, et de leurs soubresauts passés ${ }^{(*)}$. Le cycle du carbone y joue un rôle indispensable à la compréhension des évolutions, dont les paléoclimatologues identifient les traces.

(^) J. Poitou, "Composition atmosphérique et bilan radiatif ", Reflets de la physique n³3 (2013) 28-33.

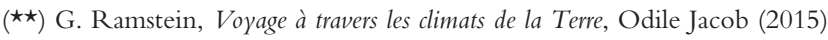

\section{Les glaciations du quaternaire} encadré 4

\section{doivent beaucoup aux rétroactions}

Depuis trois millions d'années, la Terre connaît des alternances de périodes interglaciaires (telle que la période actuelle) avec une calotte de glace sur l'Antarctique et une sur le Groenland, et des périodes glaciaires avec la formation de deux gigantesques calottes supplémentaires, I'une recouvrant le nord de l'Europe et l'autre le nord de l'Amérique.

Une entrée en glaciation est déclenchée par des conditions saisonnières particulières : des hivers relativement doux aux hautes latitudes de I'hémisphère Nord, avec des températures douces aux tropiques, pour que l'atmosphère soit assez humide pour apporter des précipitations neigeuses abondantes; l'été assez frais pour que la neige accumulée pendant I'hiver ne fonde pas totalement. Ceci requiert que le solstice d'hiver soit proche du périhélie ${ }^{(+)}$et le solstice d'été proche de l'aphélie ${ }^{(+)}$, avec une excentricité de l'orbite terrestre pas trop faible et une obliquité forte. De la neige va donc subsister toute l'année aux hautes latitudes, ce qui y accroît l'albédo, réduit l'absorption du rayonnement solaire et provoque un refroidissement. Le remplacement qui s'ensuit, de la forêt boréale par la toundra, une végétation basse, augmente l'albédo de ces régions enneigées, renforçant le refroidissement. Avec le refroidissement, la solubilité du $\mathrm{CO}_{2}$ croît fortement dans les eaux océaniques ; la circulation thermohaline va en stocker une quantité importante dans les profondeurs de l'océan, entraînant une baisse de sa concentration atmosphérique et donc une réduction importante de l'effet de serre. Parallèlement, l'activité des micro-organismes aux hautes latitudes va également baisser, et avec elle la production de méthane.

Tous ces processus de rétroaction amplifient le refroidissement. Ce sont eux qui ont permis, il y a 21000 ans, l'accumulation sur le nord de l'Europe et de l'Amérique de 42 millions de $\mathrm{km}^{3}$ de glace, abaissant de 120 mètres le niveau de l'océan mondial.

(+) Point de la trajectoire d'un objet céleste en orbite héliocentrique qui est le plus proche (périhélie) ou le plus éloigné (aphélie) de l'étoile autour de laquelle il tourne.

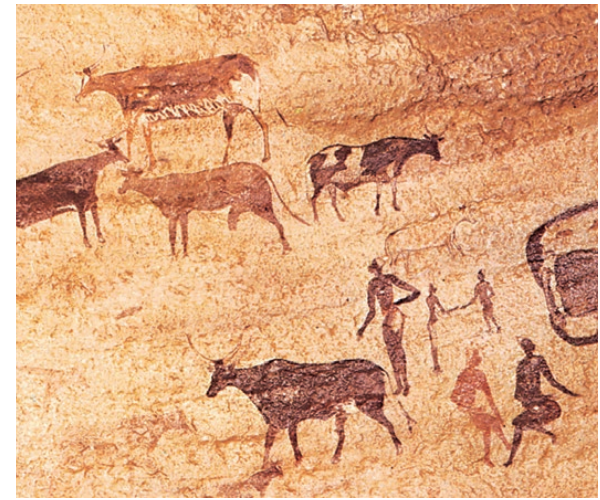

9. Peinture rupestre du Tassili, montrant l'élevage dans le Sahara « vert ", il y a 6000 ans.

solaires. Mais son rôle climatique prépondérant est d'abaisser l'effet de serre par les quantités importantes de carbone qu'elle stocke. Elle agit aussi par évapotranspiration : l'humidité que la forêt transmet à l'atmosphère (fig. 8) favorise les précipitations régionales, un phénomène qui a contribué au "Sahara vert " ily a 6000 ans (fig. 9). L'évapotranspiration plus forte agit aussi sur le bilan d'énergie en surface, avec un effet rafraîchissant.

\section{Modélisation du climat}

Terminons cette brève visite de la machine climatique par la modélisation. Les premiers modèles de climat se sont attachés à décrire le fonctionnement de l'atmosphère en termes de lois physiques du transport. Y interviennent la mécanique des fluides (loi de Navier-Stokes) dans un système en rotation, les lois de conservation (masse, énergie, quantité de mouvement) et la thermodynamique ; les forces en jeu sont le gradient de pression, la gravité, les forces de Coriolis et celles de friction. Cette composante atmosphère des premiers modèles est aussi celle de la prévision météorologique ; l'environnement (température de surface des mers, état des sols...) y est considéré comme passif.

Un tel modèle ne peut pas prendre en compte correctement les redistributions d'énergie par l'océan et l'atmosphère. Au modèle de circulation atmosphérique, on a donc couplé un modèle de circulation océanique. Une des difficultés pour réaliser ce couplage tient à la variété des temps caractéristiques des processus en jeu dans les deux milieux (fig. 6). Mais, nous l'avons vu, le climat agit sur l'environnement et celuici réagit sur le climat. Une modélisation réaliste devra faire intervenir l'ensemble 
\〉

des composantes de l'environnement. Ce n'est plus seulement un modèle de climat, mais un modèle du système Terre (fig. 10) qui est utilisé de nos jours pour décrire l'évolution du climat. Un tel modèle nécessite des ressources informatiques (puissance, temps de calcul) très importantes, qui le rendent inutilisable là où des simulations nombreuses sont nécessaires pour élucider certains processus à l'œuvre dans les climats actuels ou passés.

Ces outils sont donc complétés par une hiérarchie de modèles plus théoriques ou simplifiés, qui jouent un rôle déterminant pour comprendre les mécanismes et interactions avec l'environnement, de la circulation générale océanique et atmosphérique, et pour décrypter la variabilité naturelle du climat. Ils jouent un rôle clé pour évaluer les rétroactions et les réponses du climat à diverses perturbations du bilan radiatif terrestre. Ils sont essentiels pour comprendre les grands changements du passé, connus grâce aux données issues d'archives naturelles du climat. Ils permettent de donner sens aux changements actuels, en évaluant la part de la variabilité naturelle, spontanée, du climat, et la part de l'influence humaine. Ils sont essentiels aussi pour anticiper les risques futurs.

\section{Remerciements}

Ce document ne serait pas ce qu'il est sans les conseils avisés de Valérie Masson-Delmotte et de Pascale Braconnot.

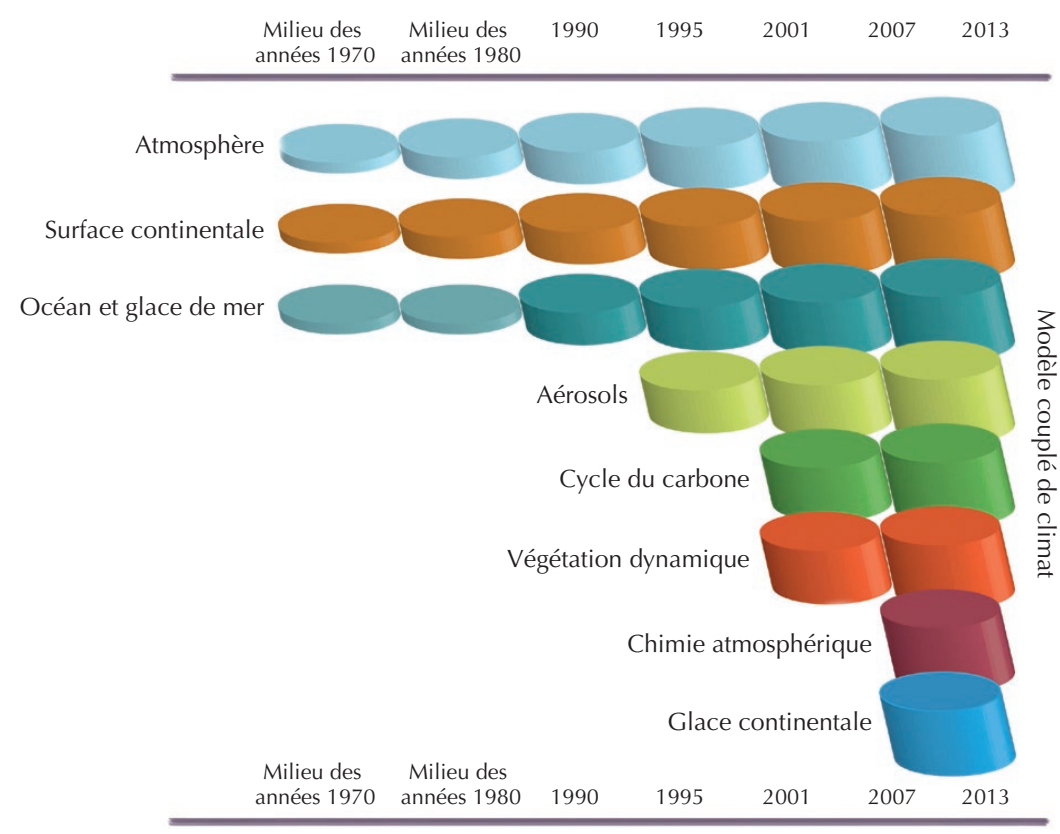

10. Évolution des modèles de climat au cours du temps. Le nombre de composantes prises en compte augmente, en même temps que le degré de raffinement de leur description (ce que traduit l'augmentation de hauteur des cylindres entre les années 1970 et 2013). Les modèles actuels aspirent à décrire l'ensemble du « système Terre ». Noter que les calottes de glace ne sont pas encore intégrées de manière standard.

\section{En savoir plus}

1• S. Weart, PNAS 110, suppl. 1 (2013) 3657-3664.

2• J. Poitou, P. Braconnot, V. Masson-Delmotte, La Terre et les hommes, EDP Sciences (2015).

3- C. Jeandel et R. Mosseri, Le climat à découvert, CNRS Éditions (2011).

4 Rapport du GIEC : Climate change 2013, The physical science basis, www.ipcc.ch/report/ars/wg1/.
5• Vidéo de la conférence scientifique "0ur Common Future under Climate Change", UNESCO, Paris, 7-10 juillet 2015 : www.youtube.com/watch?list=PLGhlfpu 6hUmltc8GHvW0jp0rdkX1HqWGX\&v=kWY6e5l1Kew
6• T. Gerkema et L. Gostiaux, « Petite histoire de la force de Coriolis", Reflets de la physique $\mathrm{n}^{\circ} \mathbf{1 7}$ (2008-2009) 18-21.

